\title{
BMJ Open Longitudinal changes in intraocular pressure and association with systemic factors and refractive error: Lingtou Eye Cohort Study
}

\author{
Xiaotong Han, ${ }^{1}$ Tangjian Yang, ${ }^{2}$ Jian Zhang, ${ }^{1}$ Sha $\mathrm{Yu},{ }^{2}$ Xinxing Guo, ${ }^{1}$ William Yan, ${ }^{3}$ \\ Yin $\mathrm{Hu},{ }^{1}$ Mingguang $\mathrm{He}^{1,3}$
}

To cite: Han X, Yang T, Zhang J, et al. Longitudinal changes in intraocular pressure and association with systemic factors and refractive error: Lingtou Eye Cohort Study. BMJ Open 2018;8:e019416. doi:10.1136/ bmjopen-2017-019416

- Prepublication history for this paper is available online. To view these files, please visit the journal online (http://dx.doi org/10.1136/bmjopen-2017019416).

Received 11 September 2017 Revised 5 January 2018 Accepted 9 January 2018
Check for updates

${ }^{1}$ State Key Laboratory of Ophthalmology, Zhongshan Ophthalmic Center, Sun Yat-sen University, Guangzhou, China ${ }^{2}$ Department of Ophthalmology, Guangzhou No. 11 People's Hospital, Guangzhou, China ${ }^{3}$ Ophthalmology, Department of Surgery, Centre for Eye Research Australia, University of Melbourne, Melbourne, Australia

Correspondence to

Dr Mingguang $\mathrm{He}$;

mingguang_he@yahoo.com

\section{ABSTRACT}

Objectives To investigate the longitudinal changes in intraocular pressure (IOP) and its associations with refractive error and systemic determinants in a Chinese geriatric population.

Design Prospective cohort study.

Setting Guangzhou Government Servant Physical Checkup Center, Guangzhou, China.

Participants 4413 government employees aged no less than 40 years ( $41.9 \%$ female) attending annual physical and eye examinations were included in this study. The inclusion criterion was having attended the 2010 follow-up examination. The exclusion criteria include glaucoma or intraocular surgery history, $10 \mathrm{P}>21 \mathrm{~mm} \mathrm{Hg}$ at any visit or without available IOP data at all visits from 2010 to 2014 .

Primary and secondary outcome measures The outcome measure was IOP at each follow-up visit from 2010 to 2014. Mixed-effect model was used to assess the relationship between longitudinal changes in IOP and potential risk factors.

Results For the 2653 participants who had available IOP data at both the 2010 and 2014 follow-up visits, the average change in IOP was an increase of $0.43(95 \% \mathrm{Cl}$ 0.36 to 0.50$) \mathrm{mm} \mathrm{Hg}$. For the whole study population and in the optimised mixed model, there was a non-linear increase of IOP with age $(\mathrm{P}<0.001)$, with greater changes in younger subjects and in women $(P<0.001$ and $P=0.002$, respectively). Elevations in systolic blood pressure, diastolic blood pressure, body mass index (BMI) and fasting plasma glucose (FPG), as well as a myopic shift (all with $\mathrm{P}<0.001$ ), during the follow-up were associated with an increasing trend of IOP, while serum lipids were found to be not significantly associated.

Conclusions In this cohort of elderly Chinese adults, IOP increases non-linearly with ageing. People with increasing blood pressure, BMI, FPG and myopic progression are more likely to have IOP elevation over time.

\section{INTRODUCTION}

Glaucoma is a leading cause of irreversible blindness globally and has been estimated to affect nearly 111.8 million people in $2040 .{ }^{1}$ Reduction of intraocular pressure (IOP) is the only proven effective treatment of

\section{Strengths and limitations of this study}

- A large number of participants, with annual measurements of intraocular pressure (IOP) and many systemic factors, were included in this study to assess the longitudinal changes in IOP and its associations with potential risk factors.

- Mixed-effect model was used to assess the change-to-change relationships after controlling for confounding factors.

- The relationship between spherical equivalent and IOP was less known in the literature and was assessed in this study.

- IOP was measured by a non-contact tonometer instead of the gold standard Goldmann tonometer.

- This cohort was not population-based, limiting the generalisability of the findings.

glaucoma, which may slow the progression of vision loss and even result in improvement of visual fields. ${ }^{2}$ Most studies have reported an increasing prevalence of glaucoma with age, but it is debatable that IOP change represents ageing or cohort effects. ${ }^{3}$ Cross-sectional and longitudinal studies on Caucasian and African populations had almost consistently shown a positive relationship between IOP and age. ${ }^{4}$ However, IOP was found to decrease with age in most cross-sectional studies on Asian population. ${ }^{5}$ Longitudinal studies in Asia were limited with inconsistent results. ${ }^{6}$

Systemic factors such as systolic blood pressure (SBP) and body mass index (BMI) have been suggested to be associated with $\mathrm{IOP}^{7}$ However, most studies were cross-sectional in design and unable to demonstrate a causal association. Although myopia was an important risk factor for primary open-angle glaucoma (POAG), the relationship between IOP and refractive error has not been clearly illustrated, and to the best of our knowledge the association between spherical equivalent (SE) and IOP had never been investigated longitudinally. 
We have previously illustrated the potential role of cohort effect on age-related IOP changes based on the Lingtou Eye Cohort Study; but the 2-year follow-up duration might be too short to establish a convincing relationship between IOP and age. ${ }^{8}$ Thus, we conducted a longitudinal analysis on the same cohort over 5 years to evaluate the effect of age, SE and related systemic risk factors on IOP.

\section{METHODS}

\section{Study population}

The Lingtou Eye Cohort Study is an ongoing prospective study with government employees attending annual physical check-up and eye examinations at the Guangzhou Government Servant Physical Check-up Center; detailed methodology can be found elsewhere. ${ }^{9}$ This cohort was originally established to investigate the associations of retinal abnormalities with systemic cardiovascular and metabolic conditions, and participants no less than 40 years of age and without history of major cardiovascular events were enrolled in 2008 for this long-term follow-up study on account of their high retention rates for annual check-up. Written informed consent was obtained from all participants.

Baseline evaluations including physical and ocular examinations were performed in 2008, as well as a brief questionnaire administered by inperson interviews. Detailed medical history, including ocular, systemic, and surgical history, was confirmed by medical records. Blood pressure (BP) was measured per standard protocol with an automatic upper-arm BP monitor (HBP-9020; OMRON, Osaka, Japan) by trained nurses. Height and weight were measured with subjects in light clothes and without shoes in standing position using an automatic height and weight scale (HNH-318; OMRON). Height was measured to the nearest $0.5 \mathrm{~cm}$ and weight was measured to the nearest $0.5 \mathrm{~kg}$. BMI was calculated as weight in kilograms divided by height in metres squared. Fasting plasma glucose (FPG), triglycerides (TG), total cholesterol (TC) and high-density lipoprotein cholesterol (HDL) were measured per standardised protocols. Automated refraction (KR-8800; Topcon, Japan) was performed in both eyes separately before pupil dilation. The mean of three consecutive measurements for spherical and cylindrical power was recorded as the final reading for each eye. SE was calculated as spherical $+1 / 2$ cylindrical power. All participants were invited to attend subsequent annual follow-up examinations. Identical examination procedures and protocols were applied throughout the study.

IOP measurement was initiated in 2010. Of all the participants who had attended the 2010 examination, we further excluded those who had undergone eye surgery in either eye or with $\mathrm{IOP}>21 \mathrm{~mm} \mathrm{Hg}$ at any visit, or cases without available IOP value at all visits. The remaining participants were the study population of the current study, and further divided into four birth cohorts based on age in 2010 , by 10-year intervals.

\section{Measurement of IOP}

Non-contact tonometer (CT-80A Computerised Tonometer, Topcon) was used to measure the IOP of both eyes before pupil dilation and was measured by a trained nurse. Three consecutive measurements were performed for each eye, and the mean was recorded as the final result if the standard error of the three measurements was less than $5 \%$. If standard error was $\geq 5 \%$ or if the subject could not cooperate, the testing was attempted two more times with a $5 \mathrm{~min}$ interval. If a standard error $<5 \%$ was not obtained on retesting, the IOP value was excluded from the analysis. One final reading was recorded for each eye. Tonometer was calibrated every 6 months by the equipment provider throughout the study.

\section{Statistical analysis}

All data analyses were performed using Stata package (Stata V.8.0). Measurements from the right eye were selected for analysis because of the high correlation between the two eyes and were summarised using the mean and SD measures. Student's t-test was used for continuous variables and $\chi^{2}$ test was used for categorical variables to compare the characteristics of participants included and not included in the analysis. Trend analysis was used to assess the trend in longitudinal changes of IOP, SE and related systemic factors with increasing baseline age, and group t-test was used to assess gender differences in longitudinal changes. Associations between longitudinal change in IOP and potential risk factors were assessed using three mixed-effect models with the assumption that data missing was random, and predictors of missing data were included in the models. Each visit from 2010 to 2014 was assigned a number from 0 to 4 accordingly and was used as a proxy for time. All of the model covariates were adjusted for baseline age and sex. Examination time, examination time squared, TC, TG, HDL, SBP, diastolic blood pressure (DBP), FPG and SE were included as fixed effects. Individual subject was considered as random effect. Mean changes and 95\% CIs were calculated from the mixed models. Model 1 was a univariate regression; model 2 was a multivariate regression; and model 3 was the optimised model after excluding the most insignificant variables from model 2 step by step. $P$ values of $<0.05$ were considered statistically significant.

\section{RESULTS}

Figure 1 presents the flow chart of the current study protocol. Of the 4882 participants who attended the 2010 follow-up examination, we further excluded 296 participants whose IOP was $>21 \mathrm{~mm} \mathrm{Hg}, 141$ who had undergone eye surgery in either eye and 32 without available IOP values at all visits. The remaining 4413 participants ( $41.9 \%$ female) were included in the analysis, with a mean age of $60.8 \pm 8.8$ years in 2010 . The mean (SD) number of visits over 5 years was 3.7 (1.5) for men and 3.5 (1.6) for women. Table 1 summarises the characteristics of the participants included and excluded from the analysis. Participants who were included were significantly younger 


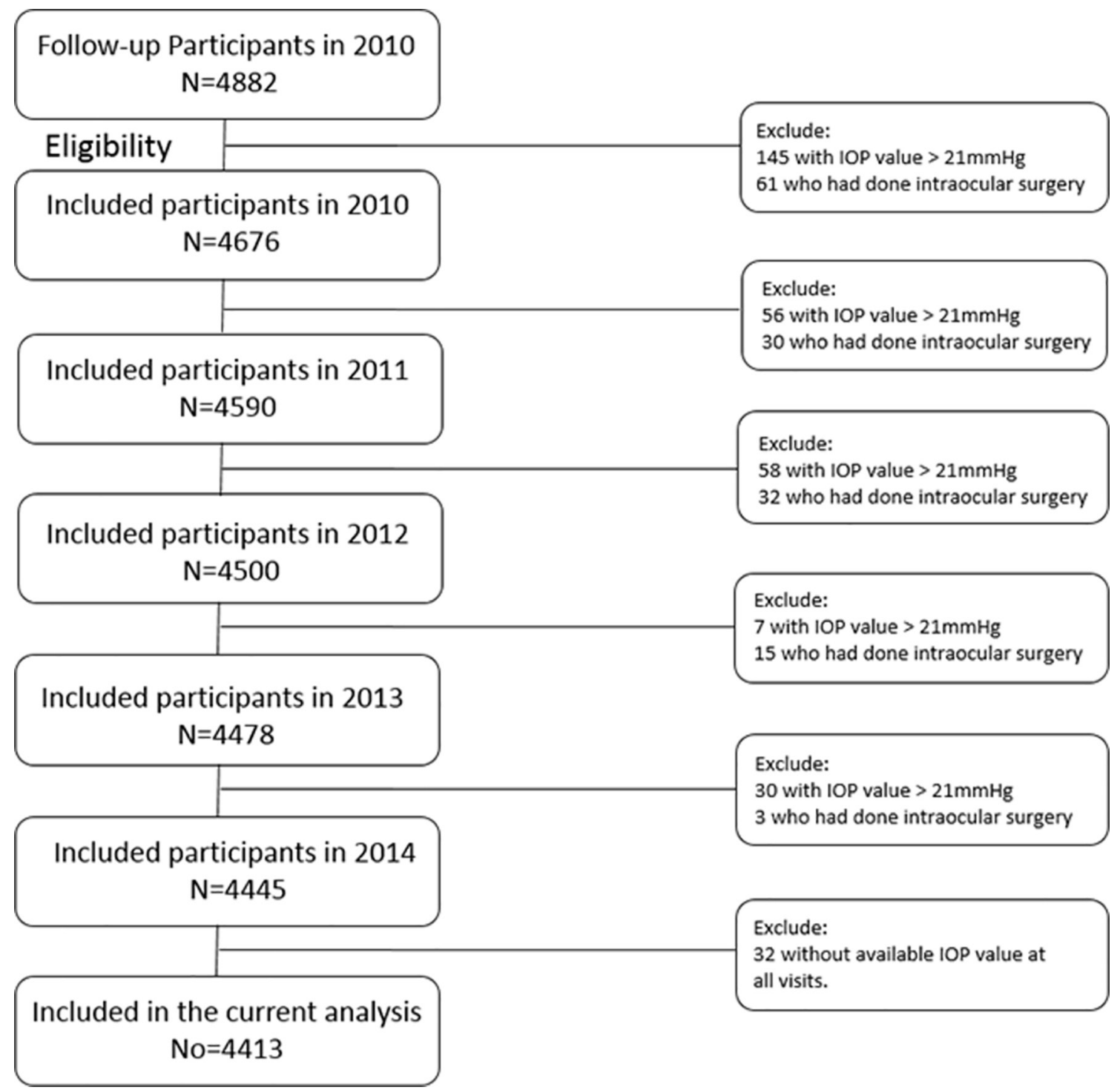

Figure 1 Flow chart of the current study. IOP, intraocular pressure.

( $\mathrm{P}<0.001)$, with lower BMI $(\mathrm{P}=0.02)$, lower $\mathrm{BP}(\mathrm{P}<0.001)$, as well as lower FPG $(\mathrm{P}<0.001)$ and IOP $(\mathrm{P}<0.001)$ values.

Table 2 shows the changes of IOP, SE and related systemic factors from 2010 to 2014 for the 2653 participants who had attended IOP measurements both in 2010 and 2014. The mean change of IOP was $0.44 \pm 0.05 \mathrm{~mm} \mathrm{Hg}$ for women and $0.51 \pm 0.04 \mathrm{~mm} \mathrm{Hg}$ for men without significant intersex differences. BMI, SBP, DBP, TC and TG decreased, while HDL and FPG increased during the follow-up period. SE showed an overall slight hyperopic shift for this subset of participants. There was a trend for older participants to have a larger decrease in SBP $(\mathrm{P}=0.004)$, TC $(\mathrm{P}=0.001)$ and $\mathrm{SE}(\mathrm{P}<0.001)$, as well as increase in FPG $(\mathrm{P}<0.001)$. Women were more likely to have HDL elevation than men $(\mathrm{P}<0.001)$.

Table 3 shows the association between longitudinal changes in IOP and related risk factors. Mixed model analysis showed a non-linear increasing trend of IOP as examination time increases $(\mathrm{P}<0.001)$. Lower baseline age $(\mathrm{P}<0.001)$, female gender $(\mathrm{P}=0.002)$, and increasing trend of SBP $(\mathrm{P}<0.001)$, DBP $(\mathrm{P}<0.001)$, BMI $(\mathrm{P}<0.001)$ and
FPG $(\mathrm{P}<0.001)$, as well as myopic trend of $\mathrm{SE}(\mathrm{P}<0.001)$, were associated with IOP elevation during the follow-up.

Table 4 shows the results of sensitivity analysis performed on the subset of participants who had attended both the 2010 and 2014 follow-up. The estimated coefficients for the longitudinal association between IOP and related parameters were similar to those of the original analysis, except for FPG, which was not statistically significant in the sensitivity analysis $(\mathrm{P}=0.07)$.

\section{DISCUSSION}

There is a non-linear increase in IOP with advancing age in our analysis. The Beijing Eye Study reported a mean change in IOP of $-1.25 \pm 2.26 \mathrm{~mm} \mathrm{Hg}$ based on single measurements from two examinations separated by a 5-year period, but the longitudinal trend of IOP change was not concluded. ${ }^{10}$ To the best of our knowledge, longitudinal association between SE and IOP had never been reported before, and we found that myopia shift was positively associated with increasing IOP. 


\begin{tabular}{lccc}
$\begin{array}{l}\text { Table } 1 \\
\text { Bnd not included in the analysis* }\end{array}$ \\
$\begin{array}{l}\text { Baseline characteristics of participants included } \\
\text { Baseline } \\
\text { characteristics }\end{array}$ & $\begin{array}{l}\text { Included } \\
(\mathbf{n}=4413)\end{array}$ & $\begin{array}{l}\text { Not included } \\
(\mathbf{n}=469)\end{array}$ & P value \\
\hline Age, years & $60.8 \pm 8.8$ & $64.5 \pm 9.0$ & $<0.001$ \\
Female, \%† & $1850(41.9 \%)$ & $173(38.9 \%)$ & 0.23 \\
BMI, kg/m ${ }^{2}$ & $24.3 \pm 3.0$ & $24.7 \pm 3.1$ & 0.02 \\
SBP, mm Hg & $127.9 \pm 17.2$ & $132.8 \pm 17.0$ & $<0.001$ \\
DBP, mm Hg & $72.1 \pm 10.7$ & $73.3 \pm 10.7$ & 0.03 \\
TC, mmol/L & $5.6 \pm 1.0$ & $5.6 \pm 1.2$ & 0.78 \\
TG, mmol/L & $1.8 \pm 1.4$ & $2.0 \pm 1.9$ & 0.17 \\
HDL, mmol/L & $1.6 \pm 0.4$ & $1.5 \pm 0.4$ & 0.17 \\
FPG, mmol/L & $5.7 \pm 1.4$ & $6.1 \pm 1.6$ & $<0.001$ \\
SE, dioptre & $-0.2 \pm 2.2$ & $-0.4 \pm 2.5$ & 0.05 \\
\hline IOP, mm Hg & $15.2 \pm 2.4$ & $18.9 \pm 4.0$ & $<0.001$ \\
\hline
\end{tabular}

Data are presented as mean \pm SD or proportions, and compared using Student's t-test unless otherwise stated.

*All participants who had attended the 2010 examination were included in the study, and participants who had undergone eye surgery in either eye or with IOP $>21 \mathrm{~mm} \mathrm{Hg}$ at any visit or without available IOP value at all visits were further excluded from the analysis.

†Comparison by $\chi^{2}$ test.

BMI, body mass index; DBP, diastolic blood pressure; FPG, fasting plasma glucose; HDL, high-density lipoprotein cholesterol; IOP, intraocular pressure; SBP, systolic blood pressure; SE, spherical equivalent; TC, total cholesterol; TG, triglyceride.

Most cross-sectional and longitudinal studies in Caucasian and African populations demonstrate a positive correlation between IOP and age, although some have shown absent or inverse associations. ${ }^{4}$ The relationship between IOP and age in Asia was more controversial given the limited amount of longitudinal studies. Nakano $e t a l^{11}$ reported IOP decreased with age in male aircraft crew members during a 10-year follow-up. This decreasing trend was supported by another 10-year ophthalmological survey and a retrospective cohort study in Japan. ${ }^{12}{ }^{13} \mathrm{~A}$ longitudinal Korean study reported an average change in IOP of $-0.065 \mathrm{~mm} \mathrm{Hg}$ per year based on a large cohort. ${ }^{14}$ However, Nomura et $a l^{6}$ reported IOP decreased with age in cross-sectional analysis but increased significantly with age in a longitudinal analysis of a large Japanese office worker population. It has been suggested that the production of aqueous humour decreases with advancing age, leading to a reduction in IOP. However, the structural changes of the trabecular meshwork increases IOP by increasing the resistance to aqueous humour outflow. ${ }^{15}$ This balance may differ between populations. In addition, difference in lifestyle and environmental factors or difference in IOP-related ocular anatomy such as central cornea thickness and anterior chamber depth may play a role in the different pattern of IOP change between studies. To be noted, existing longitudinal studies in Asia all adopted a linear assumption to estimate the association between IOP and age; however, the increasing trend of IOP with age was found to be non-linear in our analysis. Given that younger baseline age and myopic shift were shown to be significantly associated with longitudinal IOP elevation in our analysis, we speculate that the increasing trend of IOP with age is more profound in the general population than reported in the current study of participants aged 40 years or older.

Consistent with previous studies, significant associations between BP, BMI and IOP were identified in our analysis. SBP might elevate IOP in a physiological manner as higher SBP increases ocular ultrafiltration by increasing capillary pressure and decreases outflow by increasing episcleral venous pressure. The mechanism for the positive association between BMI and IOP was not fully understood, although it was suggested that increased oxidative stress due to increased adiposity leads to trabecular meshwork degeneration, as well as an increase in blood viscosity and episcleral venous pressure. ${ }^{16}$ Associations between serum lipids, blood glucose and IOP were inconclusive in the literature. A Japanese longitudinal study reported a moderately positive association, while our study found no association between longitudinal changes in serum lipids or HDL with IOP. ${ }^{13}$ The Kumejima Study and the Handan Eye Study reported a positive relationship between IOP and diabetes, but a negative relationship between IOP and haemoglobin A1c level had also been reported. ${ }^{717}$ Our study identified a positive association between changes in FPG with IOP. The osmotic gradient induced by elevated FPG levels to attract fluid and the accumulation of fibronectin in trabecular meshwork leading to increased outflow resistance, as well as diabetes-related vascular change and autonomic dysfunction, has been proposed as a possible mechanism. ${ }^{18}$

Myopia was found to be an independent risk factor for high IOP in some cross-sectional studies. ${ }^{19}$ Our study is the first to assess their relationship longitudinally and found that more myopic change was associated with an increasing trend of IOP. Previous studies consistently reported myopia as a risk factor for glaucoma, and suggested that optic nerve head and lamina cribrosa in myopic eyes appeared to be more susceptible to glaucomatous damage at any level of IOP, ${ }^{20}$ while the results of our study indicate that myopia may also increase the risk of glaucoma by increasing IOP. The Singapore Epidemiology of Eye Disease Study reported a joint effect of IOP and myopia on the risk of POAG. ${ }^{21}$ The identified positive association between myopia and IOP elevation in our study needs further validation and the underlying mechanism is unknown. We suggest that axial elongation and scleral thinning associated with myopia progression may lead to increased stress and decreased rigidity of the eyeball, thus an increasing trend of IOP. ${ }^{22}$

The gender difference in the distribution of IOP and its role in the age-related changes of IOP was inconclusive. ${ }^{23}$ Two Korean studies found a stronger decline in IOP in men, while our study found a higher increase in women. ${ }^{1424}$ The observed gender difference might be due to a higher prevalence of cardiovascular disease and smoking status in men, 


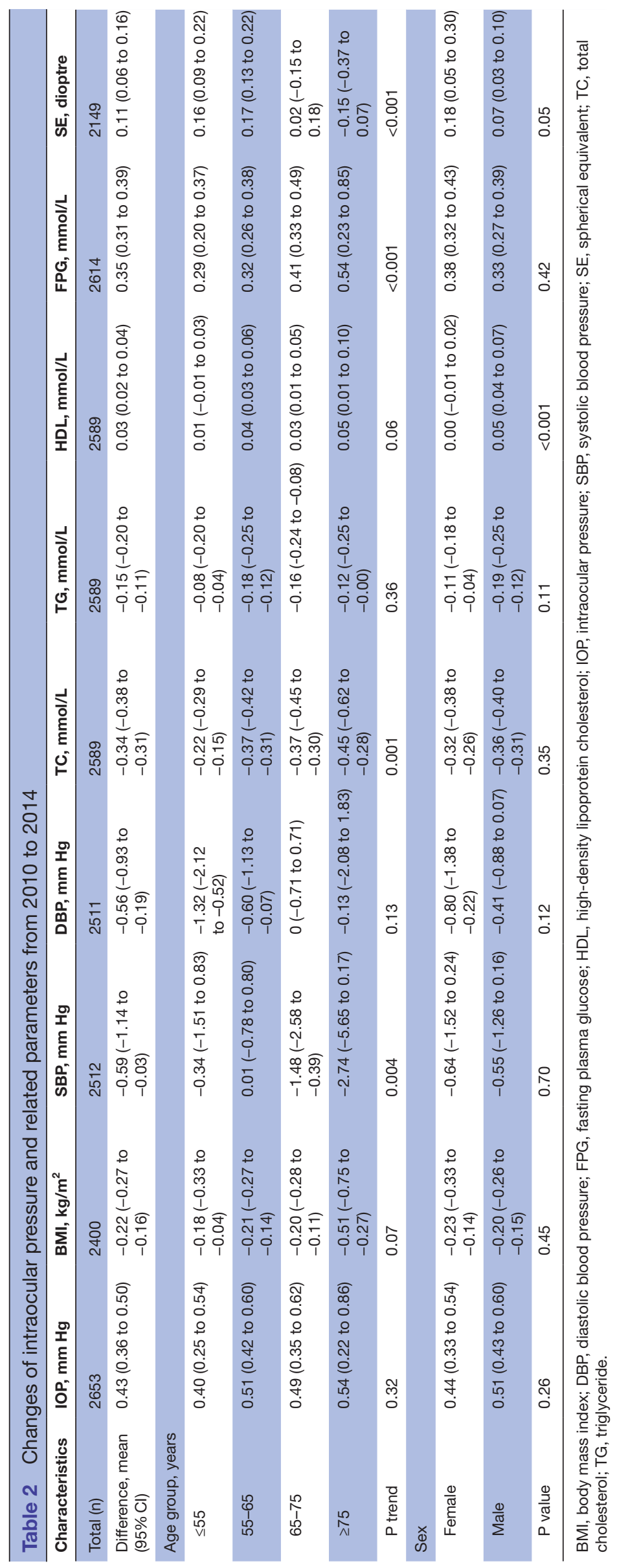


Table 3 Association between changes in intraocular pressure and other related parameters for all participants from 2010 to 2014

\begin{tabular}{|c|c|c|c|c|c|c|c|c|c|}
\hline \multirow[b]{2}{*}{ Factors } & \multicolumn{3}{|l|}{ Model 1} & \multicolumn{3}{|l|}{ Model 2} & \multicolumn{3}{|l|}{ Model 3} \\
\hline & Coefficient & $95 \% \mathrm{Cl}$ & $P$ value & Coefficient & $95 \% \mathrm{Cl}$ & $P$ value & Coefficient & $95 \% \mathrm{Cl}$ & $P$ value \\
\hline Time & -0.01 & -0.03 to 0.003 & 0.10 & -0.01 & -0.02 to 0.01 & 0.60 & -0.01 & -0.03 to 0.01 & 0.46 \\
\hline $\begin{array}{l}\text { Baseline age, } \\
\text { years }\end{array}$ & -0.21 & -0.25 to -0.17 & $<0.0001$ & -0.21 & -0.25 to -0.17 & $<0.0001$ & -0.21 & -0.25 to -0.16 & $<0.0001$ \\
\hline Gender & -0.17 & -0.30 to 0.04 & 0.01 & -0.20 & -0.34 to -0.07 & 0.003 & -0.21 & -0.35 to -0.08 & 0.002 \\
\hline DBP, mm Hg & 0.25 & 0.22 to 0.29 & $<0.0001$ & 0.15 & 0.10 to 0.20 & $<0.0001$ & 0.16 & 0.10 to 0.21 & $<0.0001$ \\
\hline $\mathrm{BMI}, \mathrm{kg} / \mathrm{m}^{2}$ & 0.07 & 0.05 to 0.09 & $<0.0001$ & 0.04 & 0.02 to 0.06 & 0.0003 & 0.04 & 0.02 to 0.06 & $<0.0001$ \\
\hline $\mathrm{TC}, \mathrm{mmol} / \mathrm{L}$ & 0.03 & -0.01 to 0.06 & 0.10 & - & - & - & - & - & - \\
\hline $\mathrm{TG}, \mathrm{mmol} / \mathrm{L}$ & 0.07 & 0.04 to 0.09 & $<0.0001$ & 0.02 & -0.01 to 0.05 & 0.28 & - & - & - \\
\hline
\end{tabular}

Model 1 is a univariate regression analysis; model 2 is a multiple regression analysis; and model 3 is the optimised model after further excluding the most insignificant variables in model 2 step by step.

BMI, blood mass index; DBP, diastolic blood pressure; FPG, fasting plasma glucose; HDL, high-density lipoprotein cholesterol; SBP, systolic blood pressure; SE, spherical equivalent; TC, total cholesterol; TG, triglyceride; time $\times$ time, examination time squared.

and hormonal difference and menopause in women. The larger skull and orbit volume in men may also contribute to the gender difference in IOP. ${ }^{25}$

Strengths of our study include a relatively large sample, the availability of annual IOP, systemic factors and SE measurements, as well as the mixed-effect model for assessing the change-to-change relationships controlled for confounding factors. However, there were some limitations. First, our study applied non-contact tonometer instead of the gold standard Goldmann tonometer to enhance participant compliance. Although there is no statistically significant difference reported between these two instruments within the normal IOP value, non-contact tonometer might have a bigger test-retest variation. ${ }^{26}$ Second, central cornea thickness is known to be associated with IOP and also changed with age but was not included in our analysis. ${ }^{27}$ Evidence suggests that the cornea stiffens with age, to which the extent that this will lead to an increase in non-contact IOP over 5

Table 4 Sensitivity analysis of the association between changes in IOP and other related parameters in participants who had undergone IOP measurement both in 2010 and 2014

\begin{tabular}{|c|c|c|c|c|c|c|c|c|c|}
\hline \multirow[b]{2}{*}{ Factors } & \multicolumn{3}{|l|}{ Model 1} & \multicolumn{3}{|l|}{ Model 2} & \multicolumn{3}{|l|}{ Model 3} \\
\hline & Coefficient & $95 \% \mathrm{Cl}$ & $P$ value & Coefficient & $95 \% \mathrm{Cl}$ & $P$ value & Coefficient & $95 \% \mathrm{Cl}$ & $P$ value \\
\hline Time & 0.001 & -0.02 to 0.02 & 0.87 & 0.01 & -0.01 to 0.03 & 0.45 & 0.01 & -0.01 to 0.02 & 0.61 \\
\hline $\begin{array}{l}\text { Baseline age, } \\
\text { years }\end{array}$ & -0.22 & -0.28 to -0.17 & $<0.0001$ & -0.22 & -0.28 to -0.17 & $<0.0001$ & -0.22 & -0.28 to -0.17 & $<0.0001$ \\
\hline Gender & -0.13 & -0.28 to 0.03 & 0.11 & -0.18 & -0.34 to -0.02 & 0.003 & -0.18 & -0.34 to -0.02 & 0.03 \\
\hline DBP, mm Hg & 0.25 & 0.21 to 0.29 & $<0.0001$ & 0.14 & 0.08 to 0.19 & $<0.0001$ & 0.14 & 0.08 to 0.20 & $<0.0001$ \\
\hline BMI, $\mathrm{kg} / \mathrm{m}^{2}$ & 0.07 & 0.05 to 0.09 & $<0.0001$ & 0.04 & 0.02 to 0.06 & 0.001 & 0.04 & 0.02 to 0.06 & 0.0003 \\
\hline $\mathrm{TC}, \mathrm{mmol} / \mathrm{L}$ & 0.02 & -0.02 to 0.06 & 0.23 & - & - & - & - & - & - \\
\hline $\mathrm{TG}, \mathrm{mmol} / \mathrm{L}$ & 0.07 & 0.04 to 0.10 & $<0.0001$ & 0.02 & -0.01 to 0.06 & 0.25 & - & - & - \\
\hline
\end{tabular}

Model 1 is a univariate regression analysis; model 2 is a multiple regression analysis; and model 3 is the optimised model after further excluding the most insignificant variables in model 2 step by step.

BMI, blood mass index; DBP, diastolic blood pressure; FPG, fasting plasma glucose; HDL, high-density lipoprotein cholesterol; IOP, intraocular pressure; SBP, systolic blood pressure; SE, spherical equivalent; TC, total cholesterol; TG, triglyceride; time $\times$ time, examination time squared. 
years and bias our result is unknown. ${ }^{28}$ Third, our study only included government employees from an annual physical check-up centre and whose IOP was less than $21 \mathrm{~mm} \mathrm{Hg}$, which may potentially limit the generalisability of our findings. Finally, as participants were not obliged to attend the annual examinations, we were unable to give the reason for dropout in this study.

In conclusion, there is a non-linear increase of IOP with age, which was more significant in women and younger subjects. Increasing BP, BMI, FPG and myopic progression were positively related to an increasing trend of IOP. Serum lipids were not found to be associated with increasing trend of IOP.

Contributors XH: data acquisition and analysis, drafting the work. TY: conception of the work, data acquisition and manuscript revision. JZ: data analysis and interpretation, manuscript revision. SY: data acquisition. XG: data acquisition and manuscript revision. WY: data interpretation and manuscript revision. YH: data acquisition and manuscript revision. MH: conception and design of the work, manuscript revision. All authors made the decision to submit this manuscript for publication, and vouch for the accuracy and completeness of the data and analyses.

Funding The study was supported by the Fundamental Research Funds of the State Key Laboratory in Ophthalmology, the National Natural Science Foundation of China (81125007), and a research grant from the Brien Holden Vision Institute. MH receives support from the University of Melbourne at Research Accelerator Program and the CERA Foundation. The Centre for Eye Research Australia receives Operational Infrastructure Support from the Victorian State Government.

Disclaimer The sponsor or funding organisation had no role in the design or conduct of this research. The sponsors had no role in study design, data collection, analysis or decision to publish, or preparation of the manuscript. The corresponding author has full access to the data and assumes final responsibility for the decision to submit for publication.

Competing interests None declared.

Patient consent Obtained.

Ethics approval The study was conducted under the guidelines of the Declaration of Helsinki and approved by the Ethics Committee of the Zhongshan Ophthalmic Center, Sun Yat-sen University.

Provenance and peer review Not commissioned; externally peer reviewed.

Data sharing statement All data relevant to this manuscript will be available upon acceptance.

Open Access This is an Open Access article distributed in accordance with the Creative Commons Attribution Non Commercial (CC BY-NC 4.0) license, which permits others to distribute, remix, adapt, build upon this work non-commercially, and license their derivative works on different terms, provided the original work is properly cited and the use is non-commercial. See: http://creativecommons.org/ licenses/by-nc/4.0/

(C) Article author(s) (or their employer(s) unless otherwise stated in the text of the article) 2018. All rights reserved. No commercial use is permitted unless otherwise expressly granted.

\section{REFERENCES}

1. Tham YC, Li X, Wong TY, et al. Global prevalence of glaucoma and projections of glaucoma burden through 2040: a systematic review and meta-analysis. Ophthalmology 2014;121:2081-90.

2. Naito T, Yoshikawa K, Mizoue S, et al. Relationship between progression of visual field defect and intraocular pressure in primary open-angle glaucoma. Clin Ophthalmol 2015;9:1373-8.

3. Chan EW, Li X, Tham YC, et al. Glaucoma in Asia: regional prevalence variations and future projections. $\mathrm{Br} \mathrm{J}$ Ophthalmol 2016;100:78-85.
4. Åström S, Stenlund $\mathrm{H}$, Lindén $\mathrm{C}$. Intraocular pressure changes over 21 years - a longitudinal age-cohort study in northern Sweden. Acta Ophthalmol 2014;92:417-20.

5. Lee MK, Cho SI, Kim H, et al. Epidemiologic characteristics of intraocular pressure in the Korean and Mongolian populations: the Healthy Twin and the GENDISCAN study. Ophthalmology 2012:119:450-7.

6. Nomura $\mathrm{H}$, Shimokata $\mathrm{H}$, Ando $\mathrm{F}$, et al. Age-related changes in intraocular pressure in a large Japanese population: a crosssectional and longitudinal study. Ophthalmology 1999;106:2016-22.

7. Tomoyose E, Higa A, Sakai H, et al. Intraocular pressure and related systemic and ocular biometric factors in a population-based study in Japan: the Kumejima study. Am J Ophthalmol 2010;150:279-86.

8. Han X, Niu Y, Guo X, et al. Age-related changes of intraocular pressure in elderly people in southern china: lingtou eye cohort study. PLoS One 2016;11:e0151766.

9. Hu Y, Niu Y, Wang D, et al. The association of longitudinal trend of fasting plasma glucose with retinal microvasculature in people without established diabetes. Invest Ophthalmol Vis Sci 2015;56:842-8.

10. Wang $Y X, X u L$, Zhang $X H$, et al. Five-year change in intraocular pressure associated with changes in arterial blood pressure and body mass index. The beijing eye study. PLoS One 2013;8:e77180.

11. Nakano T, Tatemichi M, Miura Y, et al. Long-term physiologic changes of intraocular pressure: a 10-year longitudinal analysis in young and middle-aged Japanese men. Ophthalmology 2005;112:609-16.

12. Kashiwagi K, Shibuya T, Tsukahara S. De novo age-related retinal disease and intraocular-pressure changes during a 10-year period in a Japanese adult population. Jpn J Ophthalmol 2005;49:36-40.

13. Yokomichi $\mathrm{H}$, Kashiwagi $\mathrm{K}$, Kitamura $\mathrm{K}$, et al. Evaluation of the associations between changes in intraocular pressure and metabolic syndrome parameters: a retrospective cohort study in Japan. BMJ Open 2016;6:e010360.

14. Zhao D, Kim MH, Pastor-Barriuso R, et al. A longitudinal study of age-related changes in intraocular pressure: the Kangbuk Samsung Health Study. Invest Ophthalmol Vis Sci 2014;55:6244-50.

15. Goel M, Picciani RG, Lee RK, et al. Aqueous humor dynamics: a review. Open Ophthalmol J 2010;4:52-9.

16. Zhao D, Kim MH, Pastor-Barriuso R, et al. A longitudinal study of association between adiposity markers and intraocular pressure: the kangbuk samsung health study. PLoS One 2016;11:e0146057.

17. Zhou Q, Liang YB, Wong TY, et al. Intraocular pressure and its relationship to ocular and systemic factors in a healthy Chinese rural population: the Handan Eye Study. Ophthalmic Epidemiol 2012;19:278-84.

18. Yildiz P, Kebapci MN, Mutlu F, et al. Intraocular pressure changes during oral glucose tolerance tests in diabetic and non-diabetic individuals. Exp Clin Endocrinol Diabetes 2016;124:385-8.

19. Choi JA, Han K, Park YM, et al. Age-related association of refractive error with intraocular pressure in the Korea National Health and Nutrition Examination Survey. PLoS One 2014;9:e111879.

20. Wong TY, Klein BE, Klein R, et al. Refractive errors, intraocular pressure, and glaucoma in a white population. Ophthalmology 2003;110:211-7.

21. Tham YC, Aung $T$, Fan $Q$, et al. Joint effects of intraocular pressure and myopia on risk of primary open-angle glaucoma: the singapore epidemiology of eye diseases study. Sci Rep 2016;6:19320.

22. Schmid KL, Li RW, Edwards MH, et al. The expandability of the eye in childhood myopia. Curr Eye Res 2003;26:65-71.

23. Hoehn R, Mirshahi A, Hoffmann EM, et al. Distribution of intraocular pressure and its association with ocular features and cardiovascular risk factors: the Gutenberg Health Study. Ophthalmology 2013:120:961-8

24. Baek SU, Kee C, Suh W. Longitudinal analysis of agerelated changes in intraocular pressure in South Korea. Eye 2015;29:625-9.

25. Cheng AC, Lucas PW, Yuen HK, et al. Surgical anatomy of the Chinese orbit. Ophthal Plast Reconstr Surg 2008;24:136-41.

26. Smedowski A, Weglarz B, Tarnawska D, et al. Comparison of three intraocular pressure measurement methods including biomechanical properties of the cornea. Invest Ophthalmol Vis Sci 2014;55:666-73.

27. Wang D, Huang W, Li Y, et al. Intraocular pressure, central corneal thickness, and glaucoma in chinese adults: the liwan eye study. Am J Ophthalmol 2011;152:454-62.

28. Sharifipour F, Panahi-Bazaz M, Bidar R, et al. Age-related variations in corneal biomechanical properties. J Curr Ophthalmol 2016;28:117-22. 